\title{
Postbuckling Optimization of Variable Angle Tow Composite Plates
}

\author{
Zhangming $\mathrm{Wu}^{\mathrm{a}}$, Paul M Weaver ${ }^{\mathrm{b}}$, Gangadharan Raju ${ }^{\mathrm{a}}$ \\ ${ }^{a}$ Research Assistant, Advanced Composite Centre for Innovation and Science, \\ Department of Aerospace Engineering, Queen's Building, University Walk. United \\ Kingdom \\ ${ }^{b}$ Professor in Lightweight Structures, Advanced Composite Centre for Innovation and \\ Science, Department of Aerospace Engineering, Queen's Building, University Walk. \\ United Kingdom
}

\begin{abstract}
The potential for enhanced postbuckling performance of flat plates using variable angle tow (VAT), in comparison with conventional laminated composites, has been shown previously. This paper presents an optimization strategy for the design of postbuckling behaviour of VAT composite laminates under axial compression. The postbuckling performance of composite laminated plates for a given compression loading is assessed by studying both the maximum transverse displacement and the end-shortening strain. For the postbuckling analysis of VAT composite plates, an efficient tool based on the variational principle and the Rayleigh-Ritz method is developed. In the optimization study, a mathematical definition based on Lagrangian polynomials, which requires few design parameters, is used to define a general fibre angle distribution of the VAT plate. A genetic algorithm is subsequently used to determine the optimal VAT configuration for maximum postbuckling performance. The optimization of square VAT laminates under compression loading for different in-plane boundary conditions is studied and compared with straight fibre designs.
\end{abstract}

Keywords: Postbuckling, optimization, Variable Angle Tow Plates, Variable Stiffness, Composite Laminates

\footnotetext{
*Corresponding author

Email address: paul.weaver@bristol.ac.uk (Paul M Weaver)
} 


\section{Introduction}

Use of laminated composite structures in primary load carrying aircraft structures has increased substantially over the past decade (with, for example, Airbus A380 and Boeing 787). The advent of new commercial composite manufacturing techniques such as advanced fibre placement and advanced tow placement, as well as emerging constant tow shearing [1], enable steering of tow paths in the plane of the structure and allow manufacturing of composite structures with variable stiffness. These laminates, with spatially varying fibre (tow) orientations, i.e. variable angle tow (VAT) composites, allow point-wise stiffness tailoring and have been shown to exhibit numerous structural advantages over straight fibre composites. Previous research has shown great potential of utilising VAT concept for improving structural performance, particularly the buckling and postbuckling load carrying capability of composite plates $[2,3,4,5,6]$. Despite the benefits of VAT laminates, the optimum design of such laminates is a challenge, as it involves a large number of design variables and large computational effort required by an optimization process. These challenges in the design of VAT composite plates necessitate the development of new efficient modelling and effective optimization strategies. Several optimization works on the design of VAT plates for maximum buckling load have been proposed, which are based on either using genetic algorithms (GA) [3, 7] or gradient-based mathematical programming techniques $[8,9]$. In this work, numerical studies using GA for the postbuckling design of VAT laminates under axial compression, are presented.

For the design of VAT laminates, it is both desirable and necessary to systematically represent general laminate configurations, as it reduces the number of design variables considerably. VAT laminates can be described using mathematical functions to define either the tow (fibre) trajectories, or the variation of fibre orientation angles. In the literature, existing schemes for the representation of VAT laminates are categorised into two classes. The first description is coupled with finite-element modelling, in which the design parameter in each element (node) is assumed to be constant and is allowed to independently vary across elements. For this element-based scheme, however, an additional constraint is often needed to ensure the variation of design parameters to be smooth [10].

Hyer and Lee [11] first demonstrated the use of curvilinear fibres to enhance buckling loads for a plate with a circular hole by optimizing fibre 
orientations of each element. Huang and Haftka [12] used a hybrid strategy to optimize fibre angles at each node in their finite element model for improving the strength of a laminate with a hole. More recently, Abdalla et al. [10], Setoodeh et al. [8] and IJsselmuiden et al. [9] performed the optimum design of tow-steered variable stiffness composite laminates based on weighted nodal design variables. Another way of representing VAT configurations is to employ a mathematical definition, which requires fewer design variables than the element discretization scheme and can naturally generate smooth distributions. For example, Nagendra et al. [13] proposed an optimization strategy using NURBS (Non-uniform rational B-spline) curves with a fixed number of control points to construct the curvilinear fibre paths. Honda et al. [14] defined the fibre paths using a linear combination of B-spline functions to define the fibre trajectories. Parnas et al. expressed the fibre angles in terms of either Bezier curves or cubic polynomials [15]. Olmedo and Gürdal [2] proposed a simple definition of linear variation of fibre angels using three parameters to characterise each VAT ply. The linear variation definition has been widely used for the study [16,4], test [3] and design [3] of VAT laminates. The linear fibre angle variation along a particular direction results in a limited design space, which can be further expanded by allowing the fibre orientation to vary two dimensionally [7]. The distribution of fibre angles is then expressed in a series form, for example Setoodeh et al. [17] and Alhajahmad et al. [18] employed Lobatto polynomials. A more general definition for the nonlinear variation of fibre angles using Lagrangian polynomials [7] is used in this work to establish each VAT configuration. This definition is more intuitive for the designer as the coefficient of Lagrangian polynomials is directly equal to the fibre angle at each pre-selected control point. Furthermore, the optimization results for the maximum buckling load in [7] also demonstrated that this nonlinear definition gives similar levels of fidelity and design space as finite element based models [9], yet has significantly reduced degrees of freedom.

Ghiasi et al. presented a thorough review and comparison of the optimization techniques for both constant stiffness [19] and variable stiffness [20] composite laminates. Among these techniques, the genetic algorithm (GA) is one of the most popular methods which has been extensively used in the optimization of composite laminates. Postbuckling models, either based on analytical solutions or finite element analysis (FEA), can be directly integrated into a GA to perform optimization studies, as the gradient information is not required. The GA is generally able to prevent the search 
procedure becoming trapped in local optima, provided the population size and the number of generations are sufficiently large. However, GAs also suffer from significant computational costs due to numerous evaluations of the fitness function. Kang and Kim [21] combined a GA, along with a nonlinear finite element code, to perform minimum weight optimization for composite panels subject to buckling and postbuckling design criteria. Due to the relatively poor efficiency of FEA, they implemented a parallel computing scheme with the GA to speed up the optimization procedure. Seresta et al.[22] used GA to optimize compression loaded laminated composite plates for maximum failure load in the postbuckling regime, in which the postbuckling analysis is performed by the Rayleigh-Ritz method in a semi-analytical way. More recently, Falzon and Faggiani [23] implemented an optimization procedure based on a GA, together with FEA (Abaqus), to optimize the layups of stiffened composite panels, for enhancing the damage resistance, in the postbuckling regime.

The main difficulty for the postbuckling optimization is the large computational effort involved in the process of tracing the postbuckling equilibrium path, particularly when finite element modeling is used. In the postbuckling optimization of laminated composite plates, it is often needed to determine the postbuckling behaviour of a large number of lamination configurations. Therefore, most previous works exploit analytical/semi-analytical formulations, or other approximate schemes that rapidly determine the nonlinear structural responses, so as to incorporate optimization procedures. Dickson and Biggers [24] developed a program (POSTOP) to perform the optimum design of metal or composite panels with open-section stiffeners under combined loadings. In their program, analytical/semi-analytical solutions were adopted for the buckling and postbuckling analysis and a modified feasible-direction method was applied for the optimization procedure. Shin et al. [25] presented a work on the minimum-weight optimum design of specially orthotropic laminates with respect to the postbuckling performance. The postbuckling analysis of composite laminates was performed using a Marguerre-type energy method and the phenomenon of mode jumping is considered in the optimization process. Bushnell [26] also implemented the function for the postbuckling design in his PANDA2 program, in which the postbuckling problem was solved in a semi-analytical way. In addition, an optimization strategy was introduced to retreat or remove the cases with non-convergent solutions. Other routines/tools integrated with efficient modellings were also developed and used for the postbuckling optimum design, 
such as NLPANOPT [27, 28], VICONOPT [29, 30]. As an alternative to analytical solutions, the large computational costs are reduced in some works by the application of response surface methodologies that offer a global approximation scheme to predict the postbuckling behaviour using a few number of sampled points $[31,32]$. In this paper, we have adopted a semi-analytical model derived from the mixed variational principle [6] to analyse the postbuckling behaviour of VAT plates. This model, is found to be more accurate and requires relatively less computational time, when compared with other postbuckling modelling techniques $[5,6]$ and so enables us to perform the postbuckling optimization using a genetic algorithm.

The aim of this paper is to present an optimization strategy using a GA for the design of postbuckling behaviour of VAT laminated plates and study the mechanics that are responsible for the improvement of postbuckling strength. The VAT representation, using Lagrangian polynomials to define the nonlinear distribution of fibre orientation angles across the VAT plates, is introduced in the next section. Section 3 briefly reviews the proposed semianalytical model for the postbuckling analysis of VAT plates. In section 4, the postbuckling design criteria for the optimal design of VAT plates are first presented, followed by a detailed discussion of the optimization strategy. The optimally designed VAT laminates are illustrated and discussed in section 5, compared with optimal straight-fibre laminates.

\section{Variable Angle Tow Plates}

In general, the distribution function that describes fibre orientation angles over a plate domain can be expanded using a double series form. A previously proposed scheme [7] that uses Lagrangian polynomials to define the nonlinear variation of fibre angles is adopted in this work for the optimal design of VAT laminates. In this definition, a set of $M \times N$ control points are first selected over the domain of the plate, each of which is associated with a value of fibre angle. A nonlinear distribution for the fibre orientation angles is generated using Lagrangian polynomials to interpolate the fibre angles at these control points, given by,

$$
\theta(x, y)=\sum_{m=0}^{M-1} \sum_{n=0}^{N-1} T_{m n} \cdot \prod_{m \neq i}\left(\frac{x-x_{i}}{x_{m}-x_{i}}\right) \cdot \prod_{n \neq j}\left(\frac{y-y_{j}}{y_{n}-y_{j}}\right)
$$

where $T_{m n}$ is the coefficient of each term in the series and is directly equal to the value of fibre angle at the control point $\left(x_{m}, y_{n}\right)$. Figure 1 illustrates two 
types of nonlinear variation of fibre orientation angles, one is parabolically varying along one direction and the other is varying two dimensionally. Substituting the coordinates of the selected control points into Eq. (1), explicit forms for describing the distribution of fibre orientation angles are formed. For example, the parabolic variation of fibre orientations along the $x$ direction, as shown in the left plot of Figure 1, is written as [33],

$$
\theta(\xi)=\theta(|\xi|)=T_{0}\left(2 \xi^{2}-3|\xi|+1\right)-4 T_{1}\left(\xi^{2}-|\xi|\right)+T_{2}\left(2 \xi^{2}-|\xi|\right)
$$

where $\xi=2 x / a([-1,1])$ is the normalised coordinate and $T_{0}, T_{1}, T_{2}$ are the fibre angles for $x=0, a / 4, a / 2$, respectively.

With the aid of explicit expressions, each VAT ply is parameterised in terms of the fibre orientation at the pre-selected control points, which significantly simplifies the VAT design problem. For an optimization problem, different VAT laminate configurations are constructed through varying the fibre orientations associated with these control points. Clearly, a good choice

of control points can assist an optimization procedure to rapidly achieve convergence and avoid trapping in local optima. In such circumstances, a nearly complete design space for the variable stiffness is approached by using only a few series expansion terms in Eq. (1). The selection for the number and positions of control points mainly depends on the specific problem we describe, in terms of geometry, boundary conditions and loading cases etc. It was also observed that, for a flat VAT plate, only $3 \sim 5(M$ or $N$ ) grid points along each direction are needed to achieve converged results in the buckling optimization [7]. Furthermore, this definition naturally gives a continuous, smooth distribution for the fibre orientations, which are suitable to be converted into practical tow trajectories when considering manufacturing constraints. This design scheme, that describes a VAT configuration via choosing a set of distributed control points, can also be extended for other VAT structures, such as curved panels and shells.

\section{Postbuckling Analysis}

\subsection{Basic equations}

In classical lamination plate theory (CLPT), the constitutive equations for rectangular composite plates in a partially inverted form is defined as,

$$
\left(\begin{array}{c}
\epsilon^{0} \\
\mathbf{M}
\end{array}\right)=\left[\begin{array}{cc}
\mathbf{a} & \mathbf{b} \\
-\mathbf{b}^{\mathbf{T}} & \mathbf{D}^{*}
\end{array}\right]\left(\begin{array}{c}
\mathbf{N} \\
\kappa
\end{array}\right)
$$


where $\mathbf{a}=\mathbf{A}^{-\mathbf{1}}, \mathbf{b}=-\mathbf{A}^{-\mathbf{1}} \mathbf{B}, \mathbf{D}^{*}=\mathbf{D}-\mathbf{B A}^{-\mathbf{1}} \mathbf{B}$ and $\mathbf{A}, \mathbf{B}$ and $\mathbf{D}$ are inplane, coupling and bending stiffness matrices, respectively. For VAT plates, the values of $\mathbf{a}, \mathbf{b}, \mathbf{D}$ vary with the coordinates $x$ and $y$. The term $\epsilon^{0}$ is the mid-plane strains, $\kappa$ is the curvature and $\mathbf{N}, \mathbf{M}$ are in-plane stress and bending moment resultants, respectively. As the composite plates studied herein are symmetrically laminated, there is no bending-stretching coupling and the coupling matrix $\mathbf{B}=0, \mathbf{b}=0$ and $\mathbf{D}^{*}=\mathbf{D}$.

The von Kármán large deflection equations that define the nonlinear relation between the mid-plane strains and mid-plane displacements are [34],

$$
\epsilon_{x}^{0}=u_{0, x}+\frac{1}{2} w_{, x}^{2} \quad \epsilon_{y}^{0}=v_{0, y}+\frac{1}{2} w_{, y}^{2} \quad \epsilon_{x y}^{0}=u_{0, y}+v_{0, x}+w_{, x} w_{, y}
$$

The stretching behaviour of a plate can be modelled by introducing the Airy's stress function $(\Phi)$ and the stress resultants $\mathbf{N}\left(N_{x}, N_{y}, N_{x y}\right)$ are defined as,

$$
N_{x}=\Phi_{, y y}, \quad N_{y}=\Phi_{, x x}, \quad N_{x y}=-\Phi_{, x y}
$$

For the large deflection of a composite plate, the nonlinear compatibility equation expressed in terms of Airy's stress function is given by,

$$
\begin{aligned}
& {\left[a_{11} \Phi_{, y y}+a_{12} \Phi_{, x x}-a_{16} \Phi_{, x y}\right]_{, y y}+\left[a_{12} \Phi_{, y y}+a_{22} \Phi_{, x x}-a_{26} \Phi_{, x y}\right]_{, x x}-} \\
& {\left[a_{16} \Phi_{, y y}+a_{26} \Phi_{, x x}-a_{66} \Phi_{, x y}\right]_{, x y}=\left(w_{, x y}\right)^{2}-\left(w_{, x x}\right)\left(w_{, y y}\right)}
\end{aligned}
$$

The nonlinear equilibrium equation for the large deflection is,

$$
\begin{gathered}
{\left[D_{11} w_{, x x}+D_{12} w_{, y y}+2 D_{16} w_{, x y}\right]_{, x x}+\left[D_{12} w_{, x x}+D_{22} w_{, y y}+2 D_{26} w_{, x y}\right]_{, y y}+} \\
2\left[D_{16} w_{, x x}+D_{26} w_{, y y}+2 D_{66} w_{, x y}\right]_{, x y}+\Phi_{, y y} w_{, x x}+\Phi_{, x x} w_{, y y}-2 \Phi_{, x y} w_{, x y}=0
\end{gathered}
$$

By expanding the derivatives in Eqs. (6) and (7), both the compatibility function and equilibrium equation for VAT laminates were found to involve additional higher order derivative terms with respect to the in-plane flexibility and bending stiffness coefficients $\left(a_{i j}, D_{i j}\right)[35,4,36]$, respectively.

\subsection{Semi-analytical model}

Derivation of closed-form solutions for the postbuckling analysis of VAT plates is a difficult process, due to the variable stiffness and the nonlinear behaviours. Alternatively, a semi-analytical model was developed in [6] for the postbuckling analysis of VAT composite laminates, which is applicable to 
general boundary conditions. This postbuckling model is derived based on a variational formula, which mixed the Airy's stress function and out-of-plane deflection into a single formulation, $\boldsymbol{\Pi}(\Phi, w)$. A set of nonlinear algebraic equations were obtained using the Rayleigh-Ritz method and expressed in the following tensor form [6],

$$
\begin{aligned}
& K_{p i}^{m m} \phi_{p}+K_{k i}^{m c} c_{l}+K_{k i}^{m d} d_{l}+K_{r s i}^{m b} W_{r} W_{s}=0 \\
& K_{p i}^{c m} \phi_{p}+K_{k i}^{c c} c_{l}+K_{k i}^{c d} d_{l}+K_{r s i}^{c b} W_{r} W_{s}=F x_{i} \\
& K_{p i}^{d m} \phi_{p}+K_{k i}^{d c} c_{l}+K_{k i}^{d d} d_{l}+K_{r s i}^{d b} W_{r} W_{s}=F y_{j} \\
& K_{r i}^{b b} W_{r}-K_{r p i}^{b m} W_{r} \phi_{p}-K_{r k i}^{b c} W_{r} c_{l}-K_{r k i}^{b d} W_{r} d_{l}=0
\end{aligned}
$$

where $K_{p i}^{m m}, K_{l i}^{m c}, \cdots, K_{r k i}^{b d}$ represent various stiffness matrices for a plate in the postbuckled state (refer to [6] for more details). $W_{r}\left(W_{s}\right)$ are the vectorized form of the coefficient matrix for the transverse displacement $(w)$, whilst $\phi_{p}, c_{l}, d_{l}$ are the coefficients for the series expansion of the Airy's stress function $(\Phi)$. Eq. (8) can be solved by an iterative Newton-Raphson procedure. This model offers good accuracy with reasonable computational efficiency for determining the postbuckling equilibrium paths of VAT plates, which subsequently makes the implementation of postbuckling optimization for VAT plates achievable.

\subsection{Boundary conditions}

Herein, all four edges of the plates are assumed to be simply supported. Two different in-plane boundary conditions for VAT plates under uniaxial displacement compression are studied, which are illustrated in Figure 2. The plate is subjected to uniform displacement compression $\left(x= \pm \frac{a}{2}: u=\mp \frac{\Delta_{x}}{2}\right)$, and in case $A$, the transverse edges are free to move (stress-free, $N_{y 0}=0$; and in case $B$, the transverse edges are constrained $(v=0)$.

\section{Optimum Laminate Design}

\subsection{Postbuckling optimization criteria}

The relative stiffness or a normalised postbuckling stiffness [37], however, only represents the structural strength in the postbuckling regime. In a realistic design, it often concerns load-carrying resistance over the entire range of deformation including both the prebuckling and postbuckling regimes. Therefore, it is necessary to define new quantities that assess complete structural behaviour. In this work, two quantities are used as the design criteria to 
characterise the postbuckling structural performance of laminated composite plates under a given load [38]. One is the maximum transverse deflection $w_{\max }$ and the other is the end shortening strain $\epsilon_{x}\left(=\Delta_{x} / a\right)$ along the loading direction [38]. Because the applied loading condition is constant, the overall structural strength or the load-carrying capacity directly relate to the resultant end-shortening strain or the maximum transverse deflection. Their evaluation is based on the derived postbuckling equilibrium paths, which are the nonlinear load-deflection curve and the load-end shortening curve. The loads and strains in these curves are normalised with respect to the corresponding solutions of the quasi-isotropic plate. This normalisation provides the designer with a useful measure to quantify the improvement of a trial laminate over the quasi-isotropic laminate [38]. The equivalent Young's modulus $E_{i s o}$, Poisson's ratio $\nu_{i s o}$ and bending stiffness $D_{i s o}$ of the quasi-isotropic laminate are given by [37, 38],

$$
D_{i s o}=\frac{E_{i s o} h^{3}}{12\left(1-\nu_{i s o}^{2}\right)}, \quad \nu_{i s o}=\frac{U_{4}}{U_{1}}, \quad E_{i s o}=U_{1}\left(1-\nu_{i s o}^{2}\right)
$$

where $U_{1}, U_{2}, U_{4}$ are material invariants [39].

\subsection{Failure criteria}

In this work, the optimum design of VAT plates was further investigated for their failure strength in the postbuckling regime of compression loading. The stress-based Tsai-Wu failure criterion is used herein to predict the failure of VAT laminates. For a state of plane stress, the following failure index $\left(I_{F}\right)$ according to the orthotropic Tsai-Wu criterion is defined as,

$$
I_{F}=F_{1} \sigma_{11}+F_{2} \sigma_{22}+2 F_{12} \sigma_{11} \sigma_{22}+F_{11} \sigma_{11}^{2}+F_{22} \sigma_{22}^{2}+F_{66} \sigma_{12}^{2}
$$

where $\sigma_{11}$ and $\sigma_{22}$ are the stress components along the principal material directions and $\sigma_{12}$ is the shear stress. The coefficients $F_{i j}$ in Eq. (10) are related to the material strength parameters of the lamina given by,

$$
\begin{aligned}
& F_{1}=\frac{1}{X_{t}}-\frac{1}{X_{c}}, \quad F_{2}=\frac{1}{Y_{t}}-\frac{1}{Y_{c}}, \quad F_{12}=-\frac{1}{2 \sqrt{X_{t} X_{c} Y_{t} Y_{c}}} \\
& F_{11}=\frac{1}{X_{t} X_{c}}, \quad F_{22}=\frac{1}{Y_{t} Y_{c}}, \quad F_{66}=\frac{1}{S^{2}}
\end{aligned}
$$

where $X_{t}, X_{c}, Y_{t}, Y_{c}, S$ are the experimentally determined material strength parameters. The stresses within each VAT lamina $\left(\sigma_{11}, \sigma_{22}, \sigma_{12}\right)$ are varying 
as a function of the location, which are also resulting in a spatial variation of failure indices, that is $I_{F}=I_{F}(x, y)$. The failure indices associated with each ply of the VAT plates are required to satisfy [18],

$$
I_{F}(x, y) \leq 1
$$

over the whole plate domain. The first ply failure may occur in the VAT laminate before the specified design compression load is reached and hence, it is necessary to verify the optimized VAT plates do not fail in the postbuckling regime during the optimization.

\subsection{Optimization for VAT plates}

The postbuckling response of VAT laminated composite plates depends on the laminate configuration, which refers to both the stacking sequence and the distribution of fibre orientation angles across the planform of each VAT ply. In this paper, the optimization problem for a VAT composite plate with 16 balanced, symmetric layers $\left(\left[ \pm \theta_{1} / \pm \theta_{2} / \pm \theta_{3} / \pm \theta_{4}\right]_{s}\right)$ is studied. This choice provides a reasonable design freedom for the stacking sequence, whilst the number of design parameters remains reasonable.

The design of VAT layers is based on the nonlinear variation formula Eq. (1), by which only a few parameters are needed to establish a continuously varying distribution of fibre angles. As the VAT plates studied in this paper are approximately symmetric in terms of geometry, boundary and loading conditions, only a quarter of the plate is considered for both modelling and design purposes. The control points, as shown in Figure 1, are uniformly distributed in a quarter plate domain and the distribution of fibre angles are doubly symmetric, that is $\theta(x, y)=\theta(|x|,|y|)$. The variation of fibre angles can be either varying along one direction or varying with both the $x$ and $y$ axes, $\theta_{i}(x, y)$. In the design process, the number of control points is gradually increased for the larger design space. The postbuckling optimization problem is subsequently formulated as,

Minimize:

$$
f(\mathbf{x}): \quad \epsilon_{x} / \epsilon_{x}^{i s o} \text { or } \quad w_{\max } / h
$$

Design Variables:

$$
\mathbf{x}: \quad\left[T_{00}^{(i)} \ldots T_{m n}^{(i)} \ldots T_{M N}^{(i)}\right] \quad\left(\theta_{i}, \quad i=1 \ldots 4\right)
$$

subject to:

$$
\begin{aligned}
& -\pi / 2 \leq T_{m n}^{(i)} \leq \pi / 2(i=1 \ldots 4) \\
& g_{j}(\mathbf{x}): \quad \max \left(I_{F}^{j}\left(x_{0}, y_{0}\right)\right)-1 \leq 0
\end{aligned}
$$


where $g_{j}(\mathbf{x})$ is the failure constraint for each layer of the VAT laminate. In this work, the failure indices are examined only at the top and bottom plies $(j=1,16)$ and $\left(x_{0}, y_{0}\right)$ refers to a defined grid point where the failure index are computed. The failure indices are checked at sufficient grid points to ensure no failure occurs over the domain of VAT laminate. This nonlinear constraint in a GA is commonly handled using a penalty method and the objective function is rewritten as,

$$
f(\mathbf{x})+\sum_{j} \alpha_{j} \max \left(0, g_{j}(\mathbf{x})\right)^{2}
$$

where $\alpha_{j}$ indicates the penalty coefficient corresponding to the ply failure constraint.

The end-shortening strain $\left(\epsilon_{x} / \epsilon_{x}^{i s o}\right)$ and the maximum transverse deflection $\left(w_{\max } / h\right)$ are determined from the postbuckling equilibrium curves which are obtained using the Rayleigh-Ritz model. The calculation of these two quantities is based on a fixed value of the average compression load $N_{x}$. The number of each series expansion in Eq. (8) was chosen to be 5, which offers adequate accuracy for the optimization purpose. In the procedure of tracing the postbuckling equilibrium paths of VAT laminates, mode jumping phenomena may occur. However, such consideration is out of the scope of this paper, therefore it is recommended that optimal layups obtained herein are adequately checked.

The search of optimal layups for postbuckling strength is performed using a GA. However, a sufficiently large population and generation are usually needed for the GA to achieve convergence in the postbuckling optimization of VAT plates. Based on our trial-and-error experiences, the population size was set to be at least 10 times the number of design variables, while the generation is usually set to $80 \sim 150$ depending on the population size. Several trials of the GA search with different initial populations were performed to examine the convergence of optimal results. The crossover and mutation probabilities were chosen to be 0.7 and 0.04 .

The optimization routine is first applied to find the optimal layups for constant fibre-angle laminated plates. The straight fibre laminate results are considered for assessing the improvement of structural response achieved by using the VAT concept in the postbuckling regime. Subsequently, the optimization is carried out on layups with a combination of VAT and constant fibre-angle layers. Finally, all laminae in the VAT format are considered for 
the search of optimal fibre angle distributions across the plate. This optimization study provides sufficient information to investigate both the effects of variable stiffness and stacking sequence on the postbuckling performance of VAT laminates.

\section{Results and Discussion}

This section presents the optimization results with the aim of demonstrating the benefit of VAT composite plates for postbuckling design. The study is carried out on a simply-supported square plate $(\mathrm{a}=\mathrm{b}=0.5 \mathrm{~m})$ subjected to an uniform axial compression. Two different cases (case $A$ and case $B$ ) for the in-plane boundary conditions, as illustrated in Figure 2, are considered. For each case, the postbuckling optimization is performed to minimise both the end-shortening strain and the maximum transverse displacement. Thus, four different optimization cases are implemented and carried out. The lamina properties for the graphite-epoxy composite are given by $E_{1}=163 \mathrm{GPa}, E_{2}=$ 6.8GPa, $G_{12}=3.4 \mathrm{GPa}, \nu_{12}=0.28$ and the material strength are $X_{t}=2.41 \mathrm{GPa}$, $X_{c}=1.523 \mathrm{GPa}, Y_{t}=0.0193 \mathrm{GPa}, Y_{c}=0.248 \mathrm{GPa}, S=0.107 \mathrm{GPa}$. Ply thickness is $0.13 \mathrm{~mm}$ (the plate thickness is $2.1 \mathrm{~mm}$ ).

The optimization results of these four cases are demonstrated in Tables 14. Table 1 gives the optimum laminates for minimising end-shortening strain, whereas Table 2 shows the optimum designs for minimising the maximum transverse displacement. Tables 3 and 4 list the fibre-orientations at the control points for each optimal VAT plate. The postbuckling equilibrium paths of these optimized constant stiffness and VAT laminates are compared and illustrated in Figures 3 and 4. Finite element modelling for the postbuckling analysis of VAT plates was implemented using the commercial package Abaqus to validate the optimal results given by the Rayleigh-Ritz method.

The optimal layups which give minimum end-shortening strain or the maximum transverse displacement may be different when the level of axial load $\left(N_{x 0}\right)$ is changed. The postbuckling optimization for each case is carried out with respect to several axial loads. As shown in Tables 1 and 2, the value of axial load $N_{x 0}$ is fixed to $2 N_{\text {iso }}$ and $4 N_{\text {iso }}$ for minimising the end-shortening strain and the maximum transverse displacement, respectively, in case $A$, and

to $2 N_{\text {iso }}$ for both of these two minimisation tasks in case $B$. The term $N_{\text {iso }}$ is the buckling load of the quasi-isotropic plate for the same material and boundary conditions. 
In the optimal design of straight-fibre (constant stiffness) laminates, the layer angles for the laminate configuration are restricted to the common values of $0,90,45$, and -45 deg for practical purposes [38]. From Table 1, the layup $\left[ \pm 45 / 0_{6}\right]_{s}$ gives the minimum end-shortening strain $\left(\epsilon_{x}\right)$ for both case $A$ and case $B$. The normalised end-shortening strain for a fixed axial load gives a direct measure of the effective structural stiffness, which is a function of the normalised quantities of prebuckling stiffness $\left(\bar{K}_{\text {pre }}\right)$, buckling load $\left(\bar{N}_{x}^{c r}\right)$ and postbuckling stiffness $\left(\bar{K}_{\text {post }}\right)$ given by,

$$
\frac{\epsilon_{x}}{\epsilon_{x}^{i s o}}=\frac{\bar{N}_{x}^{c r}}{\bar{K}_{\text {pre }}}+\frac{\bar{N}_{x 0}-\bar{N}_{x}^{c r}}{\bar{K}_{\text {post }}}=\frac{\bar{N}_{x 0}}{\bar{K}_{\text {post }}}-\left(\frac{1}{\bar{K}_{\text {post }}}-\frac{1}{\bar{K}_{\text {pre }}}\right) \bar{N}_{x}^{c r}
$$

From this expression (for $\bar{K}_{\text {post }}<\bar{K}_{\text {pre }}$ ) and for a given value of $\bar{N}_{x 0}$ then end-shortening strain reduces for increasing values of $\bar{K}_{\text {post }}, \bar{K}_{\text {pre }}$ and $\bar{N}_{x}^{c r}$. To minimise the end-shortening strain of a compression-loaded laminate, a large proportion of 0-deg plies in the laminate is important for carrying the compression load and also for improvement of prebuckling and postbuckling stiffness of the plate. In addition, the presence of \pm 45 -deg plies at the outer layers significantly improves the critical buckling load of the laminate. As such, the $\left[ \pm 45 / 0_{6}\right]_{s}$ layup gives the minimum end-shortening strain to this optimization problem. The laminates which have the minimum $w_{\max }$ are different for case $A$ and case $B$, but both are types of cross-ply laminate, as shown in Table 2. It was observed that the plies with 90 deg are useful for a laminate to suppress the out-of-plane deflection in the postbuckling regime. Although the value of the ply-angle is limited, these results are close to the optimal results which are determined by lamination parameters for general ply angles. Bounds of Lamination parameters facilitate the largest design space for constant thickness laminates [40]. Thus, the results of straightfibre laminates shown Tables 1 and 2 are similar to the global optima for this design problem. Consequently, these optimal straight-fibre layups can be considered as the baseline for comparison with VAT laminates.

The postbuckling behaviour of VAT plates with linear variation of fibreorientation angles $\left(\left[\phi \pm\left\langle T_{0} \mid T_{1}\right\rangle\right]_{4 s}\right)$ has been studied in previous work [6]. From this analysis, VAT plates with large buckling loads also exhibit reduced maximum transverse displacement. But, none of these linear-variation VAT plates demonstrate apparent improvement for the axial compressive stiffness in the postbuckling regime. The postbuckling optimization of VAT plates with nonlinear variation of fibre angles is now carried out in a step-by-step 
manner. The VAT plates are first constructed in a blended way, in which one or two design layers $\left(\theta_{1,2}\right)$ are of VAT format and other layers are unidirectional (straight-fibre). The fibre-orientation is initially allowed to vary along one direction. Subsequently, the laminates are configured by solely VAT layers and the fibre-orientation of each layer is allowed to vary in both $\mathrm{x}-\mathrm{y}$ directions. The number of control points is also gradually increased to provide larger design space and the convergence of optimization results with increase in control points is studied.

For case $A$, the first optimal VAT plate (\#1) shows a $16 \%$ improvement, as listed in Table 2, for minimising the end-shortening strain at the axial load $2 N_{\text {iso }}$, compared to the optimal straight-fibre laminate $\left[ \pm 45 / 0_{6}\right]_{s}$. The normalised load vs normalised end-shortening strain behaviour of these VAT laminated plates is shown in Figure 3-a. Besides the improvement for the overall axial stiffness, this VAT plate gives a $68 \%$ increase in the critical buckling load along with a $36 \%$ drop of the prebuckling stiffness. Next, the fibre-orientation $\theta(x, y)$ of the VAT plate is varied along both directions and 3-by-3 control points (design variables) are adopted to define the VAT configuration in each layer. The optimal fibre orientation at the control points are presented in Table 3. Figure 5 shows the discretised distributions for the fibre-orientation angles of $\theta_{1}$ and $\theta_{2}$ of this VAT plate. The distribution of $\theta_{3}$ and $\theta_{4}$ are similar to $\theta_{2}$. It is noted that the inner layers $\left(\theta_{2,3,4}\right)$ have a considerable proportion of 0-deg-fibre near the edges which enable the VAT plate to maintain a relatively high prebuckling stiffness. Furthermore, the fibre-orientation of the outer layers $\left(\theta_{1}\right)$ and the central region of inner layers is highly nonlinear, which gives rise to a nonuniform stress redistribution and also improvement of the buckling load and the postbuckling stiffness. From Table 2, the second optimal VAT plate (\#2) demonstrates a 36\% improvement for minimising the maximum transverse displacement over the optimal constant stiffness layup $\left[90_{4} / 0_{4}\right]_{s}$. The fibre-orientation of this VAT plate varies in the $y$ direction. The optimal VAT plate for the case where the fibre-orientation is varying with both axes is not presented, as it only has a slightly increased optimal result $\left(w_{\max } / h\right)$ over this VAT plate. The optimal fibre-orientation angles for this design (\#2) are listed in Table 3.

For case B, $13 \%$ and $37 \%$ improvements are achieved by the optimal VAT plates (\#3 and \#4) for minimising the end-shortening strain and the maximum transverse displacement, respectively. From Figure 4-a the load vs end-shorting curve, the prebuckling stiffness and buckling load of the VAT plate $(\# 3)$ is nearly the same as the $\left[ \pm 45 / 0_{6}\right]_{s}$ laminate but the postbuckling 
stiffness is significantly increased. Only the outer layers $\left( \pm \theta_{1}\right)$ for this design (\#3) has VAT orientations and the remaining inner layers are all 0-deg. The fibre-orientation of the VAT layer $\left(\theta_{1}\right)$ is only varying with respect to the $x$ direction. For this problem, no further improvements in results $\left(\epsilon_{x} / \epsilon_{x}^{i s o}\right)$ were obtained when applying the VAT configuration for the inner layers in the design or allowing the fibre-orientation to vary two dimensionally.

Figure 6 illustrates the resultant stress fields $\left(\sigma_{11}, \sigma_{22}, \sigma_{12}\right)$ and the failure indices $\left(I_{F}\right)$ at the bottom surface $(z=+h / 2)$ of the optimum VAT plate (\#1) under $7.8 \mathrm{kN}\left(2 N_{\text {iso }}\right)$ compression loading. The distribution of stress fields and failure indices obtained by the Rayleigh-Ritz method match closely with FEA. Note, the negative values of the failure indices over the plate domain are ignored and displayed as 0 . The highest failure index of this VAT plate is approximately 0.13 which is located near the centre of transverse edges. This value is small according to the Tsai-Wu failure criterion, which shows a good margin of strength safety for the optimum VAT plate. It also suggests that considering postbucking/buckling failure is more important than considering the first-ply failure in optimum design of thin-walled VAT composite laminates (at least, in the examples examined here).

Table 1: Optimum design for minimizing end-shortening strain $\epsilon_{x}$

\begin{tabular}{lcccc}
\hline \hline & $\begin{array}{c}\text { Loads } \\
\left(N_{x 0} / N_{\text {iso }}\right)\end{array}$ & $\begin{array}{c}\text { Optimum } \\
\text { Layups }\end{array}$ & $\epsilon_{x} / \epsilon_{x}^{\text {iso }}$ & Improvement $(\%)$ \\
\hline case A, Str-Fib & 2 & {$\left[ \pm 45 / 0_{6}\right]_{s}$} & 1.97 & - \\
case A, VAT & 2 & $\# 1: \mathrm{NLV}(\mathrm{x}, \mathrm{y})$ & 1.69 & 16 \\
case B, Str-Fib & 2 & {$\left[ \pm 45 / 0_{6}\right]_{s}$} & 1.68 & - \\
case B, VAT & 2 & $\# 3: \mathrm{NLV}(\mathrm{x})$ & 1.46 & 13 \\
\hline
\end{tabular}

Table 2: Optimum design for minimizing maximum transverse deflection $w_{\max }$

\begin{tabular}{lcccc}
\hline \hline & $\begin{array}{c}\text { Loads } \\
\left(N_{x 0} / N_{\text {iso }}\right)\end{array}$ & $\begin{array}{c}\text { Optimum } \\
\text { Layups }\end{array}$ & $w_{\max } / h$ & Improvement $(\%)$ \\
\hline case A, Str-Fib & 4 & {$\left[90_{4} / 0_{4}\right]_{s}$} & 2.12 & - \\
case A, VAT & 4 & $\# 2: \mathrm{NLV}(\mathrm{y})$ & 1.36 & 36 \\
case B, Str-Fib & 2.5 & {$\left[0_{2} / 90_{6}\right]_{s}$} & 1.01 & - \\
case B, VAT & 2.5 & $\# 4: \mathrm{NLV}(\mathrm{y})$ & 0.63 & 37 \\
\hline
\end{tabular}


Table 3: The fibre angles for each optimal design of VAT plate (case A)

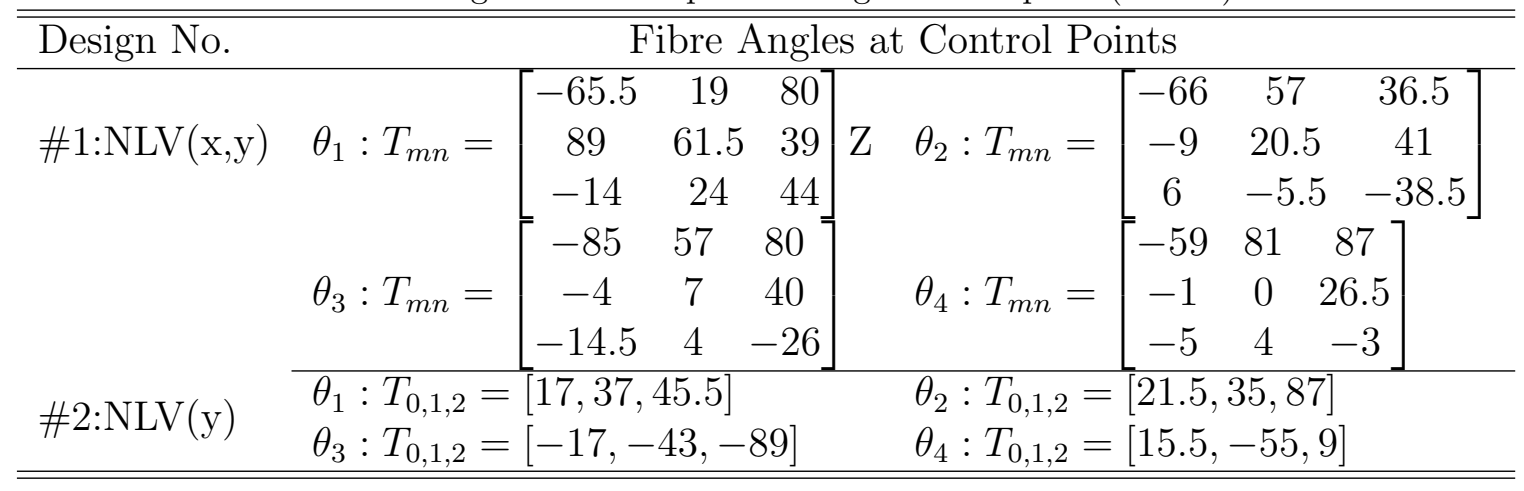

Table 4: The fibre angles for each optimal design of VAT plate (case B)

\begin{tabular}{|c|c|c|}
\hline Design No. & \multicolumn{2}{|c|}{ Fibre Angles at Control Points } \\
\hline$\# 3: \operatorname{NLV}(\mathrm{x})$ & $\theta_{1}: T_{0,1,2}=[89.5,64,23.5]$ & $\theta_{2}, \theta_{3}, \theta_{4}: 0$ \\
\hline$\# 4: \operatorname{NLV}(\mathrm{y})$ & $\begin{aligned} \theta_{1}: T_{0,1,2} & =[90,55,-20] \\
\theta_{2}: T_{0,1,2} & =[84,85,-20]\end{aligned}$ & $\theta_{3}, \theta_{4}$ \\
\hline
\end{tabular}

\section{Conclusion}

This paper presents an effective procedure for the postbuckling optimization of VAT composite plates and studies the mechanisms contributing to the improvement of the structural performance in the postbuckling regime. By relying on the simplified design scheme with efficient modelling techniques, the optimal search for benign laminate configurations was carried out using genetic algorithms. Optimal designs for the postbuckling behaviour of both the straight-fibre and VAT plates subjected to uniform axial compression under two different in-plane boundary conditions were obtained. It was noticed that, in the postbuckling regime, the optimum VAT configurations for minimising the maximum transverse displacement are similar to the optimum configuration for maximum buckling load. On the other hand, the optimum VAT configurations for minimising the end-shortening strain are different. For this case, a large proportion of 0-deg fibres is necessary for the compression-loaded VAT laminates, especially for the inner layers, to maintain a high prebuckling stiffness. In the optimal design, the VAT configurations which are placed at the outer layers further increases the buckling load, or postbuckling stiffness or indeed both. As such, the axial end-shorteng 
strain is minimised and the overall compressive stiffness is improved. It is concluded that the optimization results for the VAT laminates demonstrate substantial improvement of structural performance over constant stiffness laminates in the postbuckling regime. Further work should assess the damage tolerant viability of such laminates.

\section{Acknowledgments}

The authors wish to acknowledge EPSRC, Airbus and GKN for supporting this research under the project ABBSTRACT2 (EP/H025898/1).

\section{References}

[1] B. C. Kim, K. Potter, P. M. Weaver, Continuous tow shearing for manufacturing variable angle tow composites, Composites Part A: Applied Science and Manufacturing 43 (8) (2012) 1347 - 1356.

[2] R. Olmedo, Z. Gürdal, Buckling response of laminates with spatially varying fiber orientations, Collection of Technical Papers - AIAA/ASME Structures, Structural Dynamics and Materials Conference (1993) 2261 -2269 .

[3] B. F. Tatting, Z. Gürdal, Design and manufacture of elastically tailored tow placed plates, NASA/CR 2002- 211919 (2002) 1-14.

[4] Z. Gürdal, B. Tatting, C. Wu, Variable stiffness composite panels: Effects of stiffness variation on the in-plane and buckling response, Composites Part A: Applied Science and Manufacturing 39 (5) (2008) 911 922.

[5] T. Rahman, S. T. Ijsselmuiden, M. M. Abdalla, E. L. Jansen, Postbuckling analysis of variable stiffness composite plates using a finite elementbased perturbation method, International Journal of Structural Stability and Dynamics 11 (04) (2011) 735-753.

[6] Z. Wu, G. Raju, P. M. Weaver, Postbuckling analysis of variable angle tow composite plates., International Journal of Solids and Structures (submitted). 
[7] Z. Wu, P. M. Weaver, G. Raju, B. C. Kim, Buckling analysis and optimisation of variable angle tow composite plates, Thin-Walled Structures 60 (0) (2012) $163-172$.

[8] S. Setoodeh, M. M. Abdalla, S. T. IJsselmuiden, Z. Gürdal, Design of variable-stiffness composite panels for maximum buckling load, Composite Structures 87 (1) (2009) 109 - 117.

[9] S. T. IJsselmuiden, M. M. Abdalla, Z. Gürdal, Optimization of variablestiffness panels for maximum buckling load using lamination parameters, AIAA Journal 48 (1) (2010) 134 - 143.

[10] M. M. Abdalla, S. Setoodeh, Z. Gürdal, Design of variable stiffness composite panels for maximum fundamental frequency using lamination parameters, Composite Structures 81 (2) (2007) 283 - 291.

[11] M. Hyer, H. Lee, Use of curvilinear fiber format to improve buckling resistance of composite plates with central circular holes, Composite Structures 18 (3) (1991) 239 - 261.

[12] J. Huang, R. Haftka, Optimization of fiber orientations near a hole for increased load-carrying capacity of composite laminates, Structural and Multidisciplinary Optimization 30 (5) (2005) 335 - 341.

[13] S. Nagendra, S. Kodiyalam, J. E. Davis, V. Parthasarathy, Optimization of tow fiber paths for composite design, Collection of Technical Papers AIAA/ASME/ASCE/AHS/ASC Structures, Structural Dynamics and Materials Conference 2 (1995) 1031 - 1041.

[14] S. Honda, Y. Narita, K. Sasaki, Maximizing the fundamental frequency of laminated composite plates with optimally shaped curvilinear fibers, Journal of System Design and Dynamics 3 (6) (2009) 867-876.

[15] L. Parnas, S. Oral, U. Ceyhan, Optimum design of composite structures with curved fiber courses, Composites Science and Technology 63 (7) (2003) $1071-1082$.

[16] C. Waldhart, Z. Gürdal, C. Ribbens, Analysis of tow placed, parallel fiber, variable stiffness laminates, Collection of Technical Papers AIAA/ASME/ASCE/AHS/ASC Structures, Structural Dynamics and Materials Conference (1996) $2210-2220$. 
[17] S. Setoodeh, A. W. Blom, M. M. Abdalla, Z. Gürdal, Generating curvilinear fiber paths from lamination parameters distribution, Collection of Technical Papers - AIAA/ASME/ASCE/AHS/ASC Structures, Structural Dynamics and Materials Conference 5 (2006) 3440 - 3452.

[18] A. Alhajahmad, M. M. Abdallah, Z. Gürdal, Design tailoring for pressure pillowing using tow-placed steered fibers, Journal of Aircraft 45 (2) (2008) $630-640$.

[19] H. Ghiasi, D. Pasini, L. Lessard, Optimum stacking sequence design of composite materials part i: Constant stiffness design, Composite Structures $90(1)(2009) 1-11$.

[20] H. Ghiasi, K. Fayazbakhsh, D. Pasini, L. Lessard, Optimum stacking sequence design of composite materials. part ii: Variable stiffness design, Composite Structures 93 (1) (2010) 1 - 13.

[21] J.-H. Kang, C.-G. Kim, Minimum-weight design of compressively loaded composite plates and stiffened panels for postbuckling strength by genetic algorithm, Composite Structures 69 (2) (2005) 239 - 246.

[22] O. Seresta, M. M. Abdalla, Z. Gürdal, Optimal design of laminated composite plates for maximum post buckling strength, Collection of Technical Papers - AIAA/ASME/ASCE/AHS/ASC Structures, Structural Dynamics and Materials Conference 6 (2005) 4057 - 4068.

[23] B. Falzon, A. Faggiani, The use of a genetic algorithm to improve the postbuckling strength of stiffened composite panels susceptible to secondary instabilities, Composite Structures 94 (3) (2012) 883 - 895.

[24] S. B. Dickson, J. N.; Biggers, Postop: Postbuckled open-stiffener optimum panels - theory and capability, NASA/CR-172259 (January, 1984) $1-39$.

[25] D. K. Shin, Z. Gürdal, J. O. Hayden Griffin, Minimum weight design of laminated composite plates for postbuckling performance, Applied Mechanics Reviews 44 (11S) (1991) S219-S231.

[26] D. Bushnell, Optimization of composite, stiffened, imperfect panels under combined loads for service in the postbuckling regime, Computer 
Methods in Applied Mechanics and Engineering 103 (1-2) (1993) 43 114 .

[27] C. A. Perry, Z. Gürdal, J. H. Starnes, Minimum-weight design of compressively loaded stiffened panels for postbuckling response, Engineering Optimization 28 (3) (1997) 175-197.

[28] F. Stoll, Z. Gürdal, J. H. Starnes, A method for the geometrically nonlinear analysis of compressively loaded prismatic composite structures, NASA/CR 2002- 211919 (February 1991) 1-14.

[29] M. Lillico, R. Butler, G. Hunt, A. Watson, D. Kennedy, F. Williams, Analysis and testing of a postbuckled stiffened panel, AIAA Journal 40 (5) (2002) $996-1000$.

[30] W. Liu, R. Butler, A. Mileham, A. Green, Bilevel optimization and postbuckling of highly strained composite stiffened panels, AIAA Journal 44 (11) (2006) $2562-2570$.

[31] C. Bisagni, L. Lanzi, Post-buckling optimisation of composite stiffened panels using neural networks, Composite Structures 58 (2) (2002) 237 247.

[32] L. Lanzi, V. Giavotto, Post-buckling optimization of composite stiffened panels: Computations and experiments, Composite Structures 73 (2) (2006) $208-220$.

[33] Z. Wu, G. Raju, P. M. Weaver, Buckling analysis of vat plate using energy method, Collection of Technical Papers - 53rd AIAA/ASME Structures, Structural Dynamics and Materials Conference (2012) 1-12.

[34] P. S. Bulson, The Stability of Flat Plates, Chatto and Windus Ltd, London, 1970.

[35] Z. Gürdal, R. Olmedo, In-plane response of laminates with spatially varying fiber orientations. variable stiffness concept, AIAA journal 31 (4) (1993) $751-758$.

[36] G. Raju, Z. Wu, B. C. Kim, P. M. Weaver, Prebuckling and buckling analysis of variable angle tow plates with general boundary conditions, Composite Structures 94 (9) (2012) 2961 - 2970. 
[37] M. Pandey, A. Sherbourne, Postbuckling behaviour of optimized rectangular composite laminates, Composite Structures 23 (1) (1993) 27 38 .

[38] C. G. Diaconu, P. M. Weaver, Approximate solution and optimum design of compression-loaded, postbuckled laminated composite plates, AIAA Journal 43 (4) (2005) 906 - 914.

[39] R. M. Jones, Mechanics of composite materials, CRC Press, 2nd Revised edition edition, 1998.

[40] M. Bloomfield, C. Diaconu, P. Weaver, On feasible regions of lamination parameters for lay-up optimization of laminated composites, Proceedings of the Royal Society A: Mathematical, Physical and Engineering Sciences 465 (2104) (2009) 1123 - 1143. 

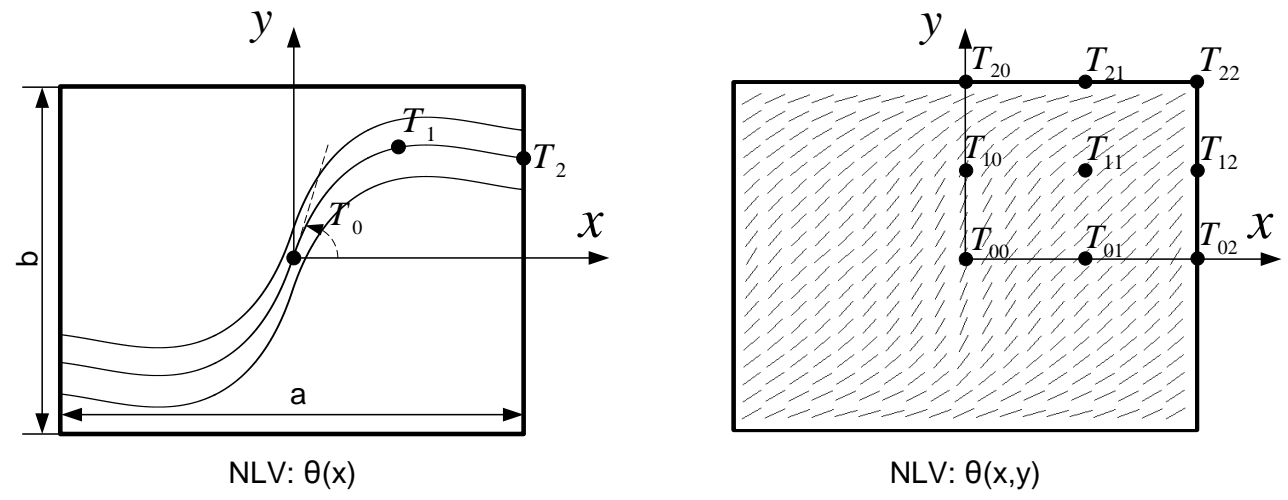

Figure 1: Two illustrations for the nonlinear variation (NLV) of fibre orientation angles over the VAT plate domain. Left: the fibre angles are parabolically varying along $x$ direction (3 control points); Right: the fibre angles are parabolically varying with both axes directions (3-by-3 control points). 


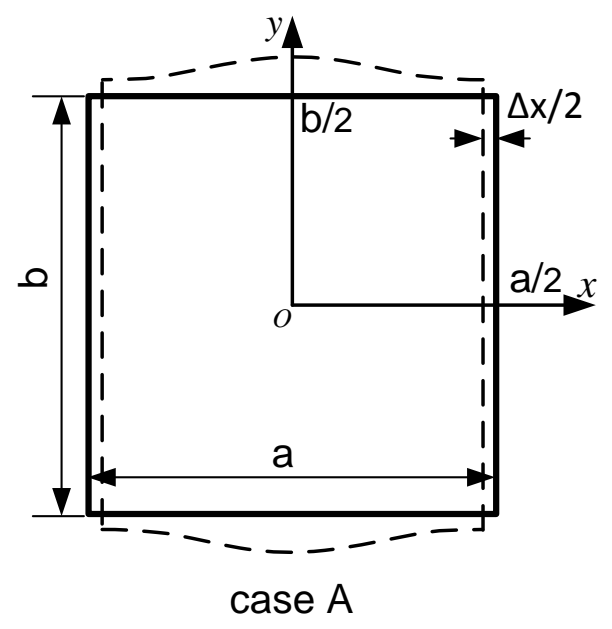

(Transverse edges free)

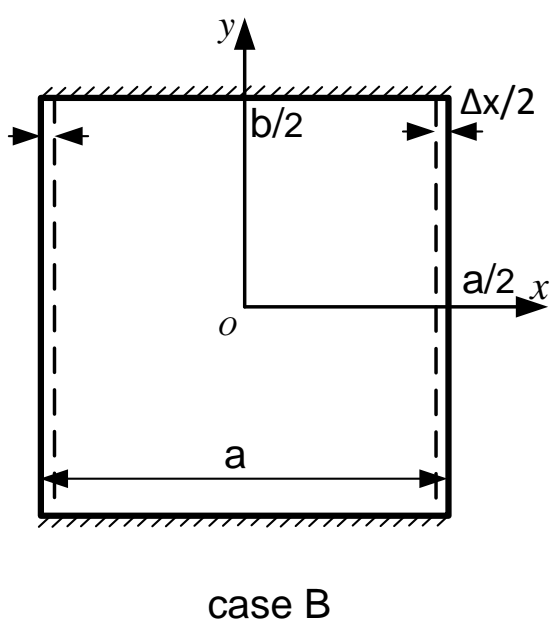

(Transverse edges constrained)

Figure 2: Geometry and boundary conditions 


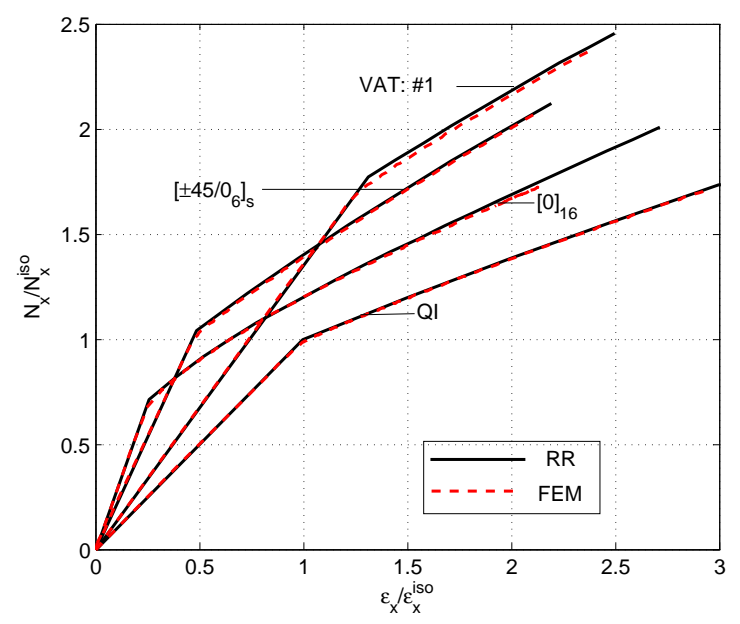

(a)

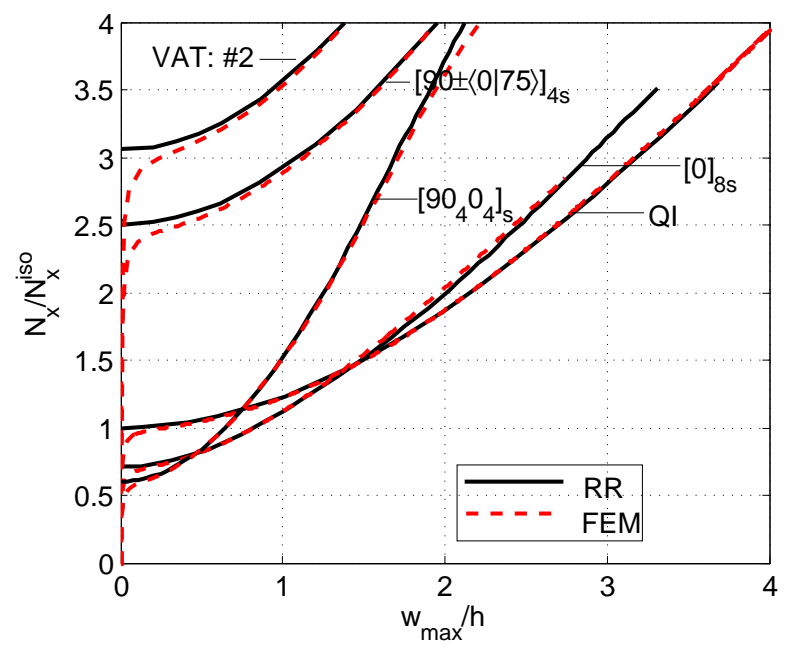

(b)

Figure 3: Rayleigh-Ritz and FEA solutions of a square simply-supported plate subjected to case $A$ : (a) Normalised axial loads $N_{x} / N_{x}^{\text {iso }}$ versus Normalised axial strain $\epsilon_{x} / \epsilon_{x}^{\text {iso }}$ (b) Normalised axial loads $N_{x} / N_{x}^{i s o}$ versus Normalized maximum transverse displacement $w_{\max } / h$ function. 


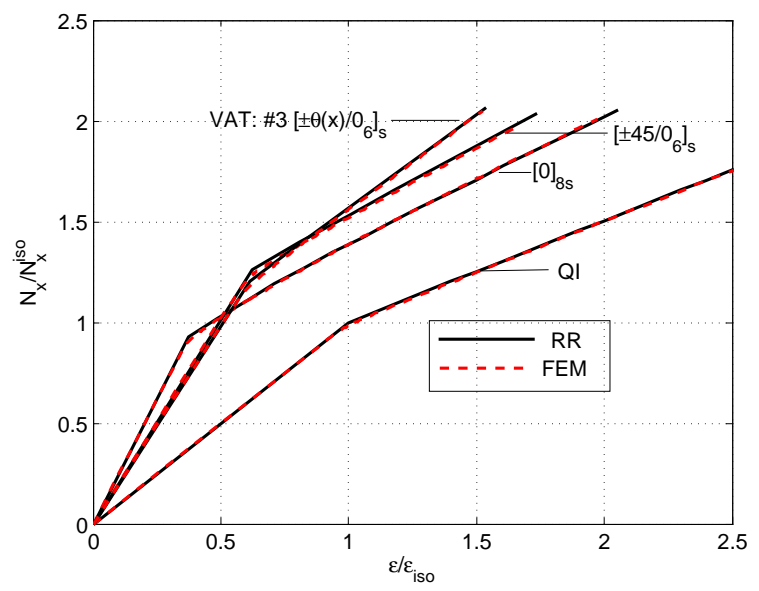

(a)

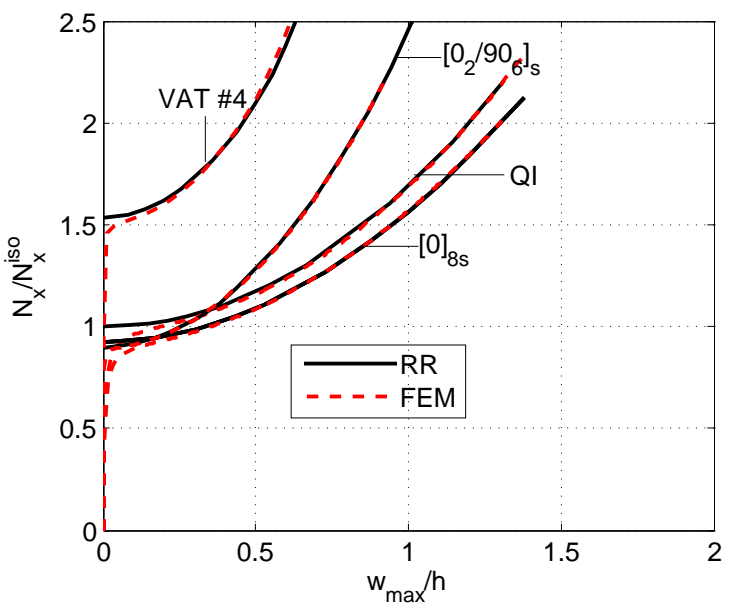

(b)

Figure 4: Rayleigh-Ritz and FEA solutions of a square simply-supported plate subjected to case B: (a) Normalised axial loads $N_{x} / N_{x}^{i s o}$ versus Normalised axial strain $\epsilon_{x} / \epsilon_{x}^{i s o}$ (b) Normalised axial loads $N_{x} / N_{x}^{i s o}$ versus Normalized maximum transverse displacement $w_{\max } / h$ function. 


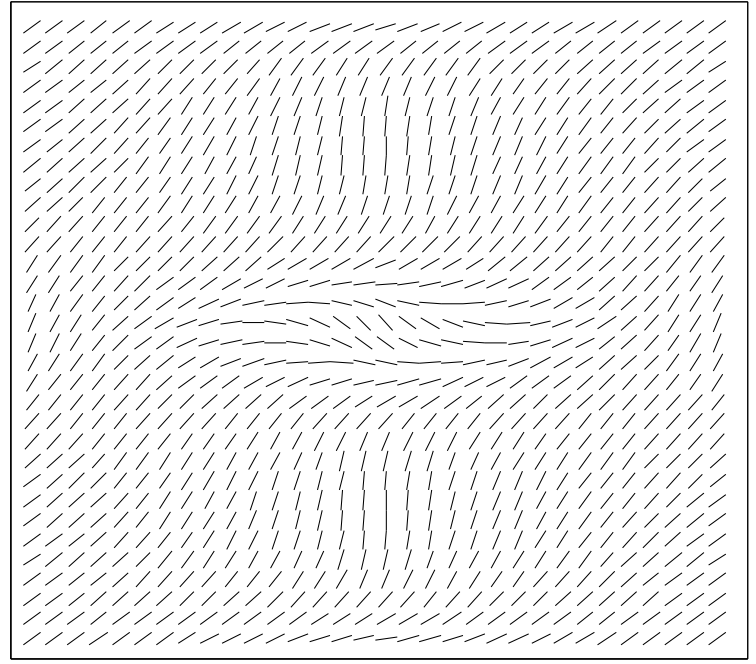

(a) $+\theta_{1}(x, y)$

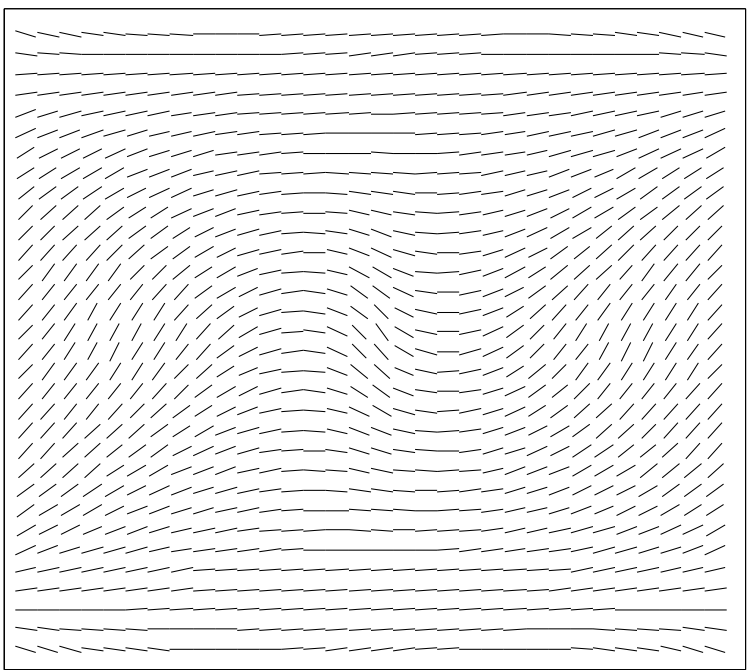

(b) $+\theta_{2}(x, y)$

Figure 5: The nonlinear variation of fibre-orientation angles for the optimum VAT plate design \#1:NLV $(\mathrm{x}, \mathrm{y})$ 

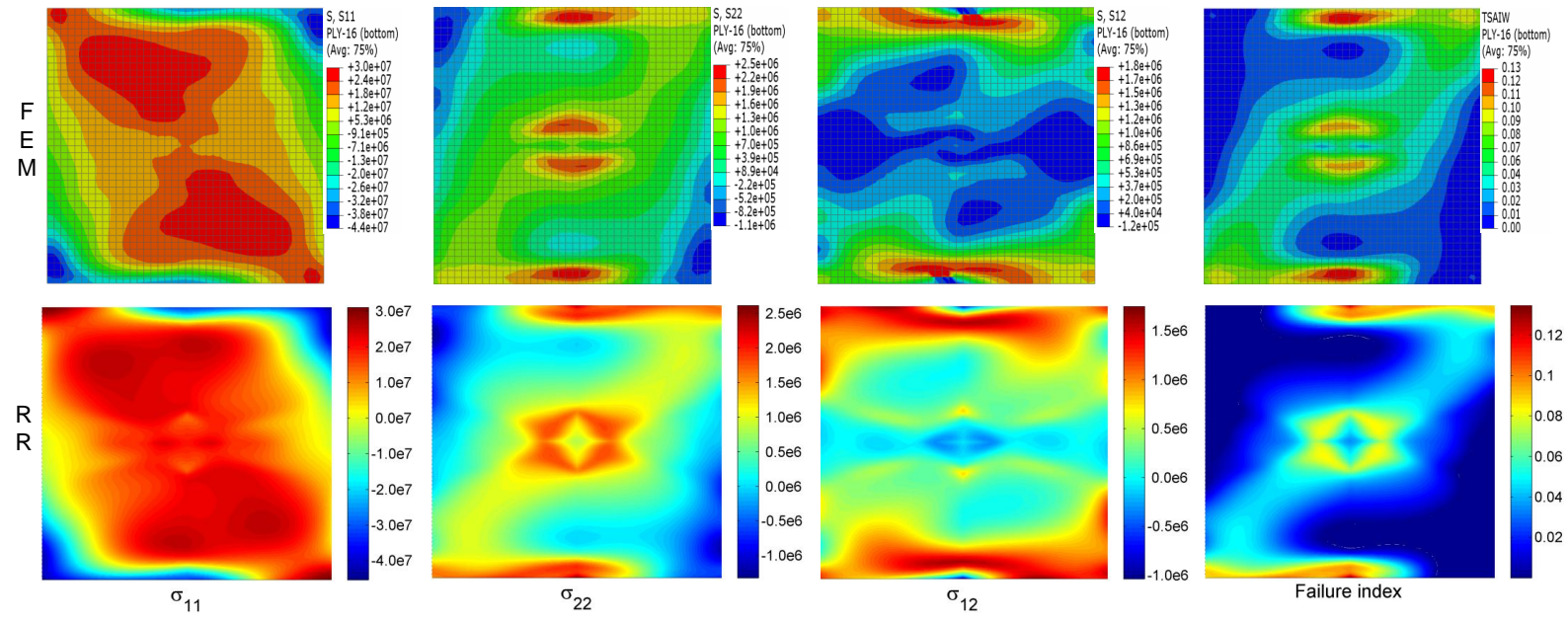

Figure 6: Comparison of FEM and Rayleigh-Ritz results on stresses and failure index of the bottom layer of the VAT plate $(\# 1)$ under $7.8 \mathrm{kN}$ compression $\left(2 N_{i s o}\right)$. 